\title{
Social capital and mental illness: a systematic review
}

\author{
Mary J De Silva, Kwame McKenzie, Trudy Harpham, Sharon R A Huttly
}

J Epidemiol Community Health 2005;59:619-627. doi: 10.1136/jech.2004.029678

See end of article for authors' affiliations ......................

Correspondence to: M De Silva, Public Health Intervention Research Unit, London School of Hygiene and Tropical Medicine, Keppel Street, London WCIE 7HT, UK; mary.desilva@|shtm.ac.uk

Accepted for publication 8 March 2005
Study objective: The concept of social capital has influenced mental health policies of nations and international organisations despite its limited evidence base. This papers aims to systematically review quantitative studies examining the association between social capital and mental illness.

Design and setting: Twenty electronic databases and the reference sections of papers were searched to identify published studies. Authors of papers were contacted for unpublished work. Anonymised papers were reviewed by the authors of this paper. Papers with a validated mental illness outcome and an exposure variable agreed as measuring social capital were included. No limitations were put on date or language of publication.

Main results: Twenty one studies met the inclusion criteria for the review. Fourteen measured social capital at the individual level and seven at an ecological level. The former offered evidence for an inverse relation between cognitive social capital and common mental disorders. There was moderate evidence for an inverse relation between cognitive social capital and child mental illness, and combined measures of social capital and common mental disorders. The seven ecological studies were diverse in methodology, populations investigated, and mental illness outcomes, making them difficult to summarise.

Conclusions: Individual and ecological social capital may measure different aspects of the social environment. Current evidence is inadequate to inform the development of specific social capital interventions to combat mental illness.
G lobal burden of disease estimates place mental illness in the top three causes of years lost due to disability. Wide variations in the rates of mental illness between geographical areas underline the need to investigate social and environmental causes. Building or sustaining healthy communities is now considered an important weapon in a state's strategy to prevent mental illness.

Despite methodological problems, the claim that social capital contributes to health inequalities within and between populations has been adopted by social scientists, policy makers, and international institutions, including the World Health Organisation and the World Bank. ${ }^{1}$ For instance, the UK government has written the building of social capital into its mental health policy. The Department of Health has explicitly cited developing social capital as an important feature of mental health promotion, ${ }^{2}$ and more recently the government's Social Exclusion Unit's action plan to improve mental health in England and Wales had advocated that authorities target access to volunteering, roles in the community, improving social networks, and general participation to improve mental health. ${ }^{3}$ It is unclear whether there is an evidence base to support these policy changes. There is a need for a systematic review of the literature to inform the debate concerning the veracity of claims that building social capital is an important facet of national mental health policy.

Social capital is a way of describing social relationships within societies or groups of people. There are many definitions $\mathrm{s}^{4-6}$ but most overlap. The most accessible definition of social capital used in the health sciences originates with Putnam. He states that social capital consists of five principal characteristics, namely: (1) community networks, voluntary, state, personal networks, and density; (2) civic engagement, participation, and use of civic networks; (3) local civic identity-sense of belonging, solidarity, and equality with other members; (4) reciprocity and norms of cooperation, a sense of obligation to help others, and confidence in return of assistance; (5) trust in the community. In this paper we use social capital to refer to features of social organisation and integration including the quantity and quality of formal and informal social interactions (often called associational life), civic participation, norms of reciprocity, and trust in others.

The theory of social capital emphasises multiple dimensions within the concept. For example, social capital can be divided into a behavioural/activity component (for example, participation) and a cognitive/perceptual component (for example, trust). These are referred to as structural and cognitive social capital respectively. ${ }^{7}$ Structural and cognitive social capital can refer to linkages and perceptions in relation to people who are similar to each other such as others in one's own community or people of the same socioeconomic status (called bonding social capital), or to people who are different, such as people outside one's community or with a different social identity (called bridging social capital). Social capital can also occur through formal institutions such as between a community and local government structures, and this is termed linking social capital. ${ }^{8}$

There has been much debate as to whether social capital should be considered a property of groups of people (an ecological construct) or also of individuals. Individual social capital is most commonly measured by asking individuals about their participation in social relationships (for example, membership of groups) and their perceptions of the quality of those relationships. For example, it may measure whether a person participates in local non-work related social groups or whether they trust their neighbours.

Ecological social capital has been most often measured by aggregating the responses of individuals in population surveys to the community level. There is, however, an acknowledged need for contextual ecological measures that do not require aggregation of individual responses or rely on individual perceptions. ${ }^{9}$ Because researchers have measured social capital both ecologically and individually and the disagreement on this subject has not as yet been resolved, ${ }^{1}$ we have reflected both streams of research in this review. 
We present a systematic review of the quantitative studies that have investigated the association between social capital and mental illness. We have collated results by type of social capital (ecological and individual, cognitive and structural) and type of mental illness. We offer the most comprehensive assessment of the evidence to date.

\section{METHODS}

\section{Search strategy}

Because of the nature of existing research on social capital, a multi-pronged approach to searching was used. Firstly, a review protocol was developed after consultation with a Cochrane information scientist. We aimed to identify all quantitative studies investigating the association between social capital and mental illness up to March 2003.

Keywords, titles, and abstracts in 20 electronic databases were searched using appropriate text words and thesaurus terms related to mental illness and social capital to identify published articles and grey literature such as reports, conferences, and theses. As different terms were, and still are, used to describe what has now been joined under the umbrella term "social capital", a wide range of search terms was used, for example, "social cohesion" and "collective efficacy" (see the appendix for a complete list of databases searched and search terms used). We did not include the search term "social support" as we are aware that there is a vast literature relating social support to mental illness, including a recent review. ${ }^{10}$ We believed that those social support studies that measure social capital should be picked up by the more specific search terms we used.

The reference sections of studies identified in this way were searched by hand. As the field is comparatively new and we believed it possible that there could be significant literature awaiting publication, the first author of all studies eligible for inclusion in the review and other authors known to work in the field were contacted in February 2003 to identify as yet unpublished research. No restrictions were put on date, geographical location, or language of publication.

\section{Selection of studies}

The abstracts of all studies were reviewed by the first author and studies potentially eligible for inclusion retrieved. Each author independently assessed each paper against agreed criteria, based only on the methods section of each paper with all references to authorship and place of publication removed. The agreed criteria were as follows: studies were included if they had a mental illness outcome (including suicide $^{11}$ ) measured using a validated tool, but excluded if they only measured sub-threshold states or mental health as compared with illness. Studies were included if they contained aspects of social capital such as membership of organisations or generalised trust in others, but did not call these terms social capital. To prevent tautological arguments, ${ }^{6}{ }^{12}$ studies were excluded if they only contained measures of social capital that could also be considered a consequence of social capital, for example neighbourhood problems such as graffiti, or homicide rates. Papers were not excluded on the grounds of methodological quality. Methodological limitations were evaluated, documented, and are presented as part of the review.

\section{Data extraction}

Effect estimates were extracted separately for studies measuring social capital at an individual and ecological level, for cognitive and structural social capital where these were measured, and for each mental illness outcome. These were later divided into childhood mental disorders, suicide, substance misuse disorders, psychotic disorders, and common mental disorder (anxiety and depression). These categories covered all the disorders that had been investigated. More than one effect estimate was available for some of the studies. For each effect estimate the most complete model was selected to achieve the most complete control for confounding. Models containing variables hypothesised to be on the causal pathway between social capital and mental illness were disregarded, and the next most complete model selected. Authors were contacted where necessary for

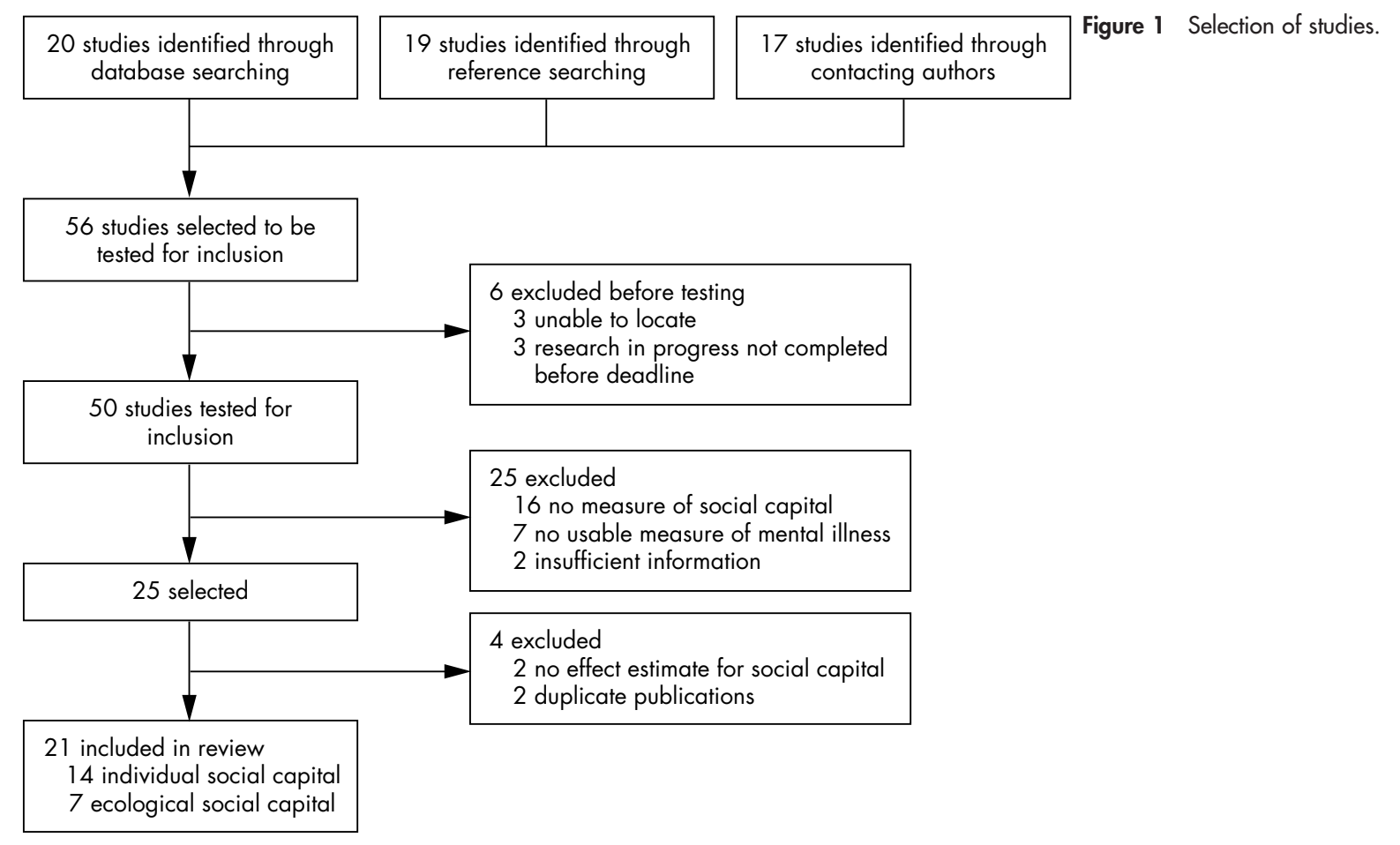


information not present in a published study that was required for full assessment of the study.

\section{Data analysis}

Differences in the measurement of social capital and mental illness, and the varied nature of the statistical techniques used by the studies made formal meta-analysis impossible. Instead, the method of grouping results developed by Ramirez et $a l^{13}$ was used. Each effect estimate was grouped into one of three categories: (1) an inverse relation between the level of social capital and the risk of mental illness significant at the 5\% level (high levels of social capital associated with lower risk of mental illness), (2) no evidence of an association (effect estimate not statistically significant at the $5 \%$ level), and (3) a positive association between the level of social capital and the rate of mental illness significant at the 5\% level (high levels of social capital associated with higher risk of mental illness). The number of studies in each category was counted, and the sample sizes aggregated. The methodological limitations of each study were evaluated using a 10 point validity checklist covering problems with the measurement of social capital, study design issues, and features of analysis that may bias the results of the study.

\section{RESULTS}

\section{Selection of studies}

Figure 1 shows the inclusion and exclusion of studies. Altogether 1693 abstracts from 20 databases were reviewed, yielding 20 studies. Nineteen additional studies were identified through reference searching, while 48 authors were contacted to identify unpublished studies. Forty one authors replied yielding a further 17 studies, making a total of 56 studies. We were unable to locate three of these studies (Albers, ${ }^{14}$ Gay and Stevenson (unpublished data), and Nation ${ }^{15}$ ) and a further three were research in progress that had not been completed (Weitzman (unpublished data), Yang et al (unpublished data), and Veenestra et al (unpublished data)). In total, 50 studies were tested for inclusion in the review.

Seven of these 50 studies were excluded as they did not have an appropriate mental illness outcome. Two measured stress (Cooper et $a l^{16}$ and Bullen (unpublished data), one posttraumatic stress but not post-traumatic stress disorder, ${ }^{17}$ one "emotional health", ${ }^{18}$ and three drug and alcohol use but not pathological use as defined by an ICD or DSM diagnosis. ${ }^{19-21}$ Sixteen studies were excluded as they did not contain an appropriate measure of social capital. Of these, one measured relationships within the family, ${ }^{22}$ and one collaboration among health care organisations, which they termed "health sector social capital" ${ }^{23}$ Seven studies only measured variables that could arguably be considered the consequence of social capital such as neighbourhood problems, ${ }^{24} 25$ or divorce or homicide rates. ${ }^{26-30}$ Seven measured concepts that may be related to social capital such as community satisfaction, ${ }^{31-35}$ sense of community, ${ }^{36}$ and unfair treatment ${ }^{37}$ but as the associations between these variables and social capital are not well demarcated these studies were excluded.

Thus 25 of the 50 studies met the inclusion criteria. Two were subsequently excluded as they used a composite score that contained among other things a measure of social capital for which we were unable to obtain a specific effect estimate. ${ }^{38} 39$ Two studies were excluded as duplicates. ${ }^{40}{ }^{41}$ This resulted in 21 studies included in the review, yielding 45 effect estimates.

\section{Description of studies}

The characteristics of the included studies measuring social capital at the individual and ecological level are shown in tables $\mathrm{IA}$ and $\mathrm{IB}$ respectively.

All but two studies were set in the developed world with $80 \%$ ( 17 of 21 ) set in the UK or USA. One third of the studies measured social capital as an ecological variable. Many different measures of social capital were used, ranging from generalised trust in others to state per capita membership of organisations. Ten studies measured both structural and cognitive aspects of social capital, nine only cognitive, and two only structural. No study explored bridging or linking dimensions of social capital, or used a contextual measure of ecological social capital.

Four studies examined mental illness in children or adolescents, the remainder examined the association in adults. Eighteen used validated psychological assessment survey tools such as GHQ-12 and CES-D, two examined completed suicide rates, and one the incidence rate of schizophrenia using case note diagnoses. In total, the 21 studies examined 17 common mental disorder outcomes, two psychotic, two substance misuse, two suicide, and nine child mental illness outcomes. Organic mental disorders, nonpsychotic disorders, and obsessive-compulsive disorders were not measured by any studies.

Tables $1 \mathrm{~A}$ and $\mathrm{IB}$ show the methodological validity of each study. All studies had a number of methodological limitations. A principal limitation was the cross sectional design of all but three studies, making the direction of the association between mental illness and social capital impossible to determine. Low levels of social capital might lead to higher rates of mental illness. It is also plausible that mental illness could weaken a person's social capital, or that geographical clustering of mental illness results in lower ecological social capital scores.

Other common methodological limitations included incomplete measurement of social capital and sampling restricted to one community type so that there was little variation in social capital scores. Although some studies were secondary analyses of survey data not specifically designed to measure social capital, these studies were in the minority.

Few studies measuring ecological social capital aggregated to the community level explored whether the geographical unit they chose represented the respondent's concept of their community. However, all but one ecological study ${ }^{61}$ measured social capital by aggregating responses from a different sample from that used to measure the mental illness outcome.

\section{Results of studies measuring social capital at the individual level}

The results of these studies are presented in table 1A, and summarised in table 2 .

\section{Cognitive social capital}

There is strong evidence of an inverse association between levels of cognitive social capital and common mental disorders, with seven of 11 effect estimates reporting higher levels of social capital to be associated with lower risk of mental illness. These effect estimates come from studies with a combined sample size seven times greater than that of those showing no association.

There is moderate evidence of an inverse association between aspects of cognitive social capital and child mental illness. Effect estimates from two studies reported a statistically significant inverse association, while an association was reported in all but one of the five non-significant effect estimates, although three of these come from the same study. ${ }^{47}$

\section{Structural social capital}

Three effect estimates showed a significant inverse association between measures of structural social capital and common mental disorder. These have a combined sample size over twice that of the seven effect estimates that do not report a significant association. One study reported a significant positive association between a person's level of group 
Table 1 (A) Characteristics of the 14 studies measuring social capital at the individual level

\begin{tabular}{|c|c|c|c|c|c|c|c|c|c|}
\hline Study ref & Setting & $\begin{array}{l}\text { Study } \\
\text { design }\end{array}$ & Population & Sample size & Validity & Social capital measure & $\begin{array}{l}\text { Mental illness } \\
\text { measure }\end{array}$ & $\begin{array}{l}\text { Statistical } \\
\text { analysis }\end{array}$ & Result \\
\hline \multirow[t]{3}{*}{ Pevalin $2003^{42} \ddagger$} & $\begin{array}{l}\text { UK national } \\
\text { sample }\end{array}$ & XS & $16+$ years & $\begin{array}{l}\text { Social participation } \\
=16750 \\
\text { Neighbourhood } \\
\text { attachment }=7,974\end{array}$ & $1,2,3$ & $\begin{array}{l}\mathrm{C}=\text { neighbourhood } \\
\text { attachment } \\
\mathrm{S}=\text { social participation }\end{array}$ & $\begin{array}{l}\text { CMD measured using } \\
\text { GHQ12 }\end{array}$ & Multivariate & $\begin{array}{l}C=-- \\
S=--\end{array}$ \\
\hline & & L & $16+$ years & $\begin{array}{l}\text { Onset }=35907 \\
\text { person years }\end{array}$ & $1,2,3$ & $\mathrm{~S}=$ social participation & $\begin{array}{l}\text { Onset, recovery } \\
\text { from, and time to } \\
\text { recovery from CMD } \\
\text { measured using } \\
\text { GHQ12 }\end{array}$ & Multivariate & $\begin{array}{l}\mathrm{S}= \\
\text { Onset = - - } \\
\text { Recovery = - } \\
\text { Time to } \\
\text { recovery = - }\end{array}$ \\
\hline & & & & $\begin{array}{l}\text { Recovery }=8840 \\
\text { person years }\end{array}$ & & & & & \\
\hline Boreham $2003^{43} \ddagger$ & $\begin{array}{l}\text { England } \\
\text { national } \\
\text { sample }\end{array}$ & XS & $16+$ years & 7988 & $2,3,10$ & $\begin{array}{l}\mathrm{C}=\text { thin trust } \\
\mathrm{S}=\text { participation in } \\
\text { organised activities }\end{array}$ & $\begin{array}{l}\text { CMD measured } \\
\text { using GHQ12 }\end{array}$ & Multilevel & $\begin{array}{l}\mathrm{C}=-- \\
\mathrm{S}= \\
\text { male }=- \\
\text { female }=--\end{array}$ \\
\hline Steptoe $2001^{44 *}$ & $\begin{array}{l}\text { England } \\
\text { Urban }\end{array}$ & XS & $\begin{array}{l}\text { Adults } \\
\text { (age n.s.) }\end{array}$ & 658 & $2,3,5,7,9$ & $C=$ social cohesion & $\begin{array}{l}\text { CMD measured using } \\
\text { GHQ12 }\end{array}$ & Multivariate & $C=--$ \\
\hline Ellaway $2001^{45 *}$ & $\begin{array}{l}\text { Scotland } \\
\text { Urban }\end{array}$ & XS & $25-65$ years & $\begin{array}{l}592 \text { adults } \\
\text { from four } \\
\text { neighbourhoods }\end{array}$ & $2,3,9$ & $\begin{array}{l}\mathrm{C}=\text { social cohesion- } \\
\text { neighbourhood attraction, } \\
\text { psychological sense of } \\
\text { community and } \\
\text { neighbouring behaviour }\end{array}$ & $\begin{array}{l}\text { CMD measured using } \\
\text { GHQ12 }\end{array}$ & Multivariate & $C=--$ \\
\hline Runyan $1998^{46 *}$ & USA & XS & $2-5$ years & 667 & 3,6 & $\begin{array}{l}C=\text { neighbourhood } \\
\text { support }\end{array}$ & $\begin{array}{l}\text { Child development = } \\
\text { BDST } \\
\text { Child behaviour = CBCL } \\
\text { combined into "child } \\
\text { doing well" yes/no }\end{array}$ & Multivariate & $C=-$ \\
\hline \multirow[t]{4}{*}{ Aneshensel $1996^{47 *}$} & $\begin{array}{l}\text { USA } 1 \\
\text { county }\end{array}$ & XS & $12-17$ years & $\begin{array}{l}877 \text { adolescents } \\
\text { from } 49 \text { census } \\
\text { tracts }\end{array}$ & $2,3,9$ & $\begin{array}{l}\mathrm{C}=\text { social cohesion }- \\
\text { whether kids and adults } \\
\text { know each other, whether } \\
\text { people are friendly }\end{array}$ & Depression $=\mathrm{CDI}$ & Multivariate & $\begin{array}{l}\mathrm{C}= \\
\text { Depression = } \\
-- \\
\text { Anxiety = - }\end{array}$ \\
\hline & & & & & & & Anxiety $=$ Hopkins & & $\mathrm{ODD}=-$ \\
\hline & & & & & & & Symptom Checklist. & & $\begin{array}{l}\text { Conduct } \\
\text { disorder }=+\end{array}$ \\
\hline & & & & & & & $\begin{array}{l}\text { Oppositional defiant } \\
\text { disorder and conduct } \\
\text { disorder = Stony } \\
\text { Brook Child Psychiatric } \\
\text { Checklist-3R }\end{array}$ & & \\
\hline Ross $2000^{48 *}$ & USA 1 county & XS & $18+$ years & 2470 & $2,3,5$ & $\begin{array}{l}\text { Informal social ties with } \\
\text { neighbours }\end{array}$ & $\begin{array}{l}\text { CMD measured using } \\
\text { Modified CES-D }\end{array}$ & Multilevel & -- \\
\hline Mitchell $2002^{49 *}$ & $\begin{array}{l}\text { USA Urban } \\
\text { poor }\end{array}$ & XS & $\begin{array}{l}\text { Adults } \\
\text { (age NS) }\end{array}$ & 222 & $3,6,10$ & $\begin{array}{l}\mathrm{C}=\text { trust, bridging ties } \\
\text { with dissimilar people } \\
\mathrm{S}=\text { group membership }\end{array}$ & $\begin{array}{l}\text { CMD measured using } \\
\text { modified } \\
\text { CES-D }\end{array}$ & Multivariate & $\begin{array}{l}\mathrm{C}=- \\
\mathrm{S}=++\end{array}$ \\
\hline Brown $1992^{50 *}$ & USA Urban & XS & $\begin{array}{l}\text { Adult African- } \\
\text { American } \\
\text { (age NS) }\end{array}$ & 927 & $2,3,6$ & $\begin{array}{l}\mathrm{S}=\text { membership of } \\
\text { voluntary associations }\end{array}$ & $\begin{array}{l}\text { CMD measured using } \\
\text { CES-D }\end{array}$ & Multivariate & $S=-$ \\
\hline Dumont $2002^{51} \S$ & $\begin{array}{l}\text { USA Urban } \\
\text { poor }\end{array}$ & XS & $\begin{array}{l}\text { Adult African- } \\
\text { American and } \\
\text { Latino women } \\
\text { (age NS) }\end{array}$ & $\begin{array}{l}397 \text { women from } \\
317 \text { census block } \\
\text { groups }\end{array}$ & $2,3,6,9,10$ & $\begin{array}{l}\mathrm{C}=\text { social cohesion- } \\
\text { neighbourhood attraction } \\
\text { and sense of community }\end{array}$ & $\begin{array}{l}\text { CMD measured using } \\
\text { CES-D }\end{array}$ & Multivariate & $C=--$ \\
\hline Dumont $2002^{52} \S$ & $\begin{array}{l}\text { USA Urban } \\
\text { poor }\end{array}$ & XS & $\begin{array}{l}\text { African- } \\
\text { American and } \\
\text { Latino children } \\
\text { aged } 7-10\end{array}$ & $\begin{array}{l}131 \text { children from } \\
121 \text { census block } \\
\text { groups }\end{array}$ & $2,3,6,9$ & $\begin{array}{l}\mathrm{C}=\text { social cohesion- } \\
\text { neighbourhood } \\
\text { attraction and sense of } \\
\text { community }\end{array}$ & $\begin{array}{l}\text { DISC } 2.3 \text { (mother's } \\
\text { report) internalising } \\
\text { and externalising } \\
\text { symptoms }\end{array}$ & Multivariate & $\begin{array}{l}\mathrm{C}= \\
\text { Internalising = } \\
- \\
\text { Externalising } \\
=--\end{array}$ \\
\hline O'Brien $1996^{53 *}$ & Russia Rural & XS & $\begin{array}{l}482 \text { heads of } \\
\text { households } \\
\text { (age NS) }\end{array}$ & $\begin{array}{l}482 \text { adults from } 3 \\
\text { villages }\end{array}$ & $2,3,9$ & $\begin{array}{l}\text { Community involvement } \\
\text { and how well "fit" into } \\
\text { village (integration) }\end{array}$ & $\begin{array}{l}\text { CMD measured using } \\
\text { modified CES-D }\end{array}$ & Multivariate & -- \\
\hline \multirow[t]{2}{*}{ Thomas $2002^{54} \S$} & $\begin{array}{l}\text { South Africa } \\
\text { Urban poor }\end{array}$ & XS & $\begin{array}{l}\text { Women under } \\
40 \text { years }\end{array}$ & 265 & $2,3,6,8$ & $\begin{array}{l}\mathrm{C}=\text { feeling part of } \\
\text { neighbourhood } \\
\mathrm{S}=\text { joining together } \\
\text { with others to address } \\
\text { a common issue }\end{array}$ & $\begin{array}{l}\text { CMD measured using } \\
\text { SRQ20 }\end{array}$ & Multivariate & $\begin{array}{l}C=- \\
S=\end{array}$ \\
\hline & $\begin{array}{l}\text { Zambia } \\
\text { Urban poor }\end{array}$ & & & 266 & & $\begin{array}{l}C=\text { feeling part of } \\
\text { neighbourhood, } \\
\text { perception of } \\
\text { neighbourhood spirit } \\
S=\text { group membership }\end{array}$ & $\begin{array}{l}\text { CMD measured using } \\
\text { SRQ20 }\end{array}$ & Multivariate & $\begin{array}{l}C=-- \\
S=-\end{array}$ \\
\hline Harpham $2002^{55_{*}}$ & $\begin{array}{l}\text { Columbia } \\
\text { Urban poor }\end{array}$ & XS & $15-25$ years & 1060 & $3,6,9$ & $\begin{array}{l}\mathrm{C}=\text { thin trust, thick trust, } \\
\text { cohesion, social support, } \\
\text { informal social control } \\
\mathrm{S}=\text { civic participation }\end{array}$ & $\begin{array}{l}\text { CMD measured using } \\
\text { SRQ20 }\end{array}$ & Multivariate & $\begin{array}{l}C=. \\
S=.\end{array}$ \\
\hline
\end{tabular}

NS, age not specified. Key to type of study: *study published in peer reviewed journal or accepted for publication, funpublished manuscript, ‡report/book chapter, §PhD thesis. Study design: XS, cross sectional; L, longitudinal; E, ecological. Social capital measure: C, cognitive social capital; S, structural social capital. Mental illness measure: CMD, common mental disorders. Key to methodological limitations: Measurement of social capital-1, secondary analysis of survey questions not originally designed to measure social capital; 2 , not all aspects of social capital measured, or combined different aspects of social capital into one score; 3, no information in validity of social capital measure; 4, clusters may not represent respondents' views of their community. Methodological limitations of study that may bias results -5 , testing the relation between social capital and mental illness not a stated objective of the study; 6 , sampled from one community type so little variation in social capital scores between individuals; 7 , response rate less than $60 \%$. Features of analysis that may bias results -8 , no control for confounding by socioeconomic status; 9 , hierarchical data structure (individual and community level variables, or clustered data), but only single level modelling used; 10, neighbourhood disorder, violence or psychological resources adjusted for. These variables may be on the causal pathway between social capital and mental illness, thus making the relation non-significant. Key to results: ++ , positive association between social capital and mental illness significant at $p<0.05 ;+$, positive association between social capital and mental illness not significant at $p=0.05 ;--$, inverse association between social capital and mental illness significant at $p<0.05$; - , inverse association between social capital and mental illness not significant at $p=0.05$; ., non-significant association, direction of association not known. 
Table 1 (B) Characteristics of the seven studies measuring social capital at the ecological level

\begin{tabular}{|c|c|c|c|c|c|c|c|c|c|}
\hline Study ref & Setting & $\begin{array}{l}\text { Study } \\
\text { design }\end{array}$ & Population & Sample size & Validity & Social capital measure & $\begin{array}{l}\text { Mental illness } \\
\text { measure }\end{array}$ & $\begin{array}{l}\text { Statistical } \\
\text { analysis }\end{array}$ & Results \\
\hline $\begin{array}{l}\text { Stafford } \\
2003^{56} \S\end{array}$ & $\begin{array}{l}\text { England, } \\
\text { Scotland } \\
\text { National sample }\end{array}$ & XS & $16+$ years & $\begin{array}{l}9223 \text { adults from } \\
239 \text { electoral } \\
\text { wards/postcode } \\
\text { sectors }\end{array}$ & 2,4 & $\begin{array}{l}\mathrm{C}=\text { trust, attachment to } \\
\text { neighbourhood, reliance } \\
\text { on neighbours for } \\
\text { practical help } \\
\mathrm{S}=\text { participation in } \\
\text { organised activities } \\
\text { Aggregated to electoral } \\
\text { ward level }\end{array}$ & $\begin{array}{l}\text { CMD measured } \\
\text { using GHQ12 }\end{array}$ & Multilevel & $\begin{array}{l}\mathrm{C}= \\
\text { Trust = + } \\
\text { Neighbourhood } \\
\text { attachment = + } \\
\text { Help from } \\
\text { neighbours = - } \\
\mathrm{S}=-\end{array}$ \\
\hline \multirow[t]{2}{*}{$\begin{array}{l}\text { Boydell } \\
2002^{5 / *}\end{array}$} & England Urban & E & All ages & $\begin{array}{l}\text { Population from } \\
\text { two electoral } \\
\text { wards }\end{array}$ & $2,3,4,7,8$ & $C=$ social cohesion & $\begin{array}{l}\text { Psychotic: } \\
\text { standardised } \\
\text { incidence rates of } \\
\text { schizophrenia } \\
\text { made by clinical } \\
\text { teams }\end{array}$ & Bi-variate & $C=--$ \\
\hline & & & & & & $\begin{array}{l}\text { Aggregated to electoral } \\
\text { ward level }\end{array}$ & & & \\
\hline \multirow[t]{2}{*}{$\begin{array}{l}\text { Drukker } \\
2003^{58_{*}}\end{array}$} & $\begin{array}{l}\text { Netherlands } \\
\text { Urban }\end{array}$ & XS & $10-12$ years & $\begin{array}{l}576 \text { from } 36 \\
\text { neighbourhoods }\end{array}$ & $2,3,7$ & $\begin{array}{l}\mathrm{C}=\text { social cohesion and } \\
\text { trust. }\end{array}$ & $\begin{array}{l}\text { Child Health } \\
\text { Questionnaire } \\
\text { mental illness and } \\
\text { behaviour } \\
\text { subscales }\end{array}$ & Multilevel & $\begin{array}{l}C= \\
\text { Mental illness = + } \\
\text { Behaviour }=+\end{array}$ \\
\hline & & & & & & $\begin{array}{l}\text { Aggregated to } \\
\text { neighbourhood level }\end{array}$ & & & \\
\hline $\begin{array}{l}\text { Harper } \\
2003+\end{array}$ & USA & $E$ & All ages & $\begin{array}{l}\text { Total population of } \\
311 \text { metropolitan } \\
\text { areas }\end{array}$ & $1,2,3,4$ & $\begin{array}{l}\mathrm{S}=\text { number of } \\
\text { membership } \\
\text { organisations per capital } \\
\text { Aggregated to } \\
\text { metropolitan area level }\end{array}$ & $\begin{array}{l}\text { Death rate from } \\
\text { suicide }\end{array}$ & Multilevel & $\begin{array}{l}\mathrm{S}= \\
\text { Males }=++ \\
\text { Females }=+\end{array}$ \\
\hline $\begin{array}{l}\text { Desai } \\
2003^{5 \% *}\end{array}$ & USA & $\mathrm{L}$ & $\begin{array}{l}\text { Patients } \\
\text { discharged from } \\
\text { a veterans' } \\
\text { psychiatric } \\
\text { inpatient program } \\
\text { (age NS) }\end{array}$ & 121933 & $1,3,4,8$ & $\begin{array}{l}\text { Level of community } \\
\text { organisational life, } \\
\text { engagement in public } \\
\text { affairs, community } \\
\text { volunteerism, informal } \\
\text { sociability, social trust. } \\
\text { Aggregated to state level }\end{array}$ & $\begin{array}{l}\text { Death from } \\
\text { suicide within } \\
\text { one year of } \\
\text { discharge }\end{array}$ & Multilevel & -- \\
\hline \multirow[t]{2}{*}{$\begin{array}{l}\text { Rosenheck } \\
2001^{00_{*}}\end{array}$} & USA Urban & $\mathrm{L}$ & $\begin{array}{l}\text { Homeless } \\
\text { people with } \\
\text { severe mental } \\
\text { illness } \\
\text { (age NS) }\end{array}$ & $\begin{array}{l}3293 \text { respondents' } \\
\text { from } 18 \\
\text { communities }\end{array}$ & $1,2,3$ & $\begin{array}{l}\text { Thin trust, volunteering, } \\
\text { community project } \\
\text { involvement, club } \\
\text { meetings attended, } \\
\text { and proportion of adults } \\
\text { who voted Aggregated } \\
\text { to county level }\end{array}$ & $\begin{array}{l}\text { CMD and } \\
\text { psychotic } \\
\text { problems } \\
\text { measured using } \\
\text { C-DIS-R and PERI. }\end{array}$ & Multilevel & $C M D=+$ \\
\hline & & & & & & & $\begin{array}{l}\text { Alcohol and drug } \\
\text { problems assessed } \\
\text { Addiction Severity } \\
\text { Index }\end{array}$ & & $\begin{array}{l}\text { Alcohol }=++ \\
\text { Drug }=+\end{array}$ \\
\hline \multirow[t]{2}{*}{$\begin{array}{l}\text { Cutrona } \\
2000^{61_{*}}\end{array}$} & USA & XS & $\begin{array}{l}\text { African } \\
\text { American } \\
\text { women, primary } \\
\text { caregiver for a } \\
10-12 \text { year old } \\
\text { child (age NS) }\end{array}$ & $\begin{array}{l}700 \text { women } \\
\text { from } 31 \text { clusters } \\
\text { created from } 259 \\
\text { block census } \\
\text { areas }\end{array}$ & $2,3,4,10$ & $\mathrm{C}=$ social cohesion & $\begin{array}{l}C M D \text { measured } \\
\text { using Mini-MASQ }\end{array}$ & Multilevel & $C=-$ \\
\hline & & & & & & Aggregated to cluster level & & & \\
\hline
\end{tabular}

NS, age not specified. Key to type of study: *study published in peer reviewed journal or accepted for publication, tunpublished manuscript, ‡report/book chapter, §PhD thesis. Study design: XS, cross sectional; L, longitudinal; E, ecological. Social capital measure: C, cognitive social capital; S, structural social capital. Mental illness measure: CMD, common mental disorders. Key to methodological limitations: Measurement of social capital-1, secondary analysis of survey questions not originally designed to measure social capital; 2, not all aspects of social capital measured, or combined different aspects of social capital into one score; 3 , no information in validity of social capital measure; 4 , clusters may not represent respondents' views of their community. Methodological limitations of study that may bias results -5 , testing the relation between social capital and mental illness not a stated objective of the study; 6 , sampled from one community type so little variation in social capital scores between individuals; 7 , response rate less than $60 \%$. Features of analysis that may bias results - 8 , no control for confounding by socioeconomic status; 9, hierarchical data structure (individual and community level variables, or clustered data), but only single level modelling used; 10, neighbourhood disorder, violence or psychological resources adjusted for. These variables may be on the causal pathway between social capital and mental illness, thus making the relation non-significant. Key to results: ++, positive association between social capital and mental illness significant at $p<0.05 ;+$, positive association between social capital and mental illness not significant at $p=0.05$; -- , inverse association between social capital and mental illness significant at $p<0.05$; - , inverse association between social capital and mental illness not significant at $p=0.05$; , non-significant association, direction of association not known.

participation and their risk of suffering from a common mental disorder. ${ }^{49}$

\section{Combined}

In two studies the social capital measurement could not be disaggregated. Both show a significant inverse association with common mental disorders. ${ }^{48} 53$

\section{Results of studies measuring social capital at the ecological level}

The results of these studies are presented in table 1B, and summarised in table 3 .
The small number of studies measuring social capital at an ecological level makes the assessment of associations between social capital and mental illness problematic. For all mental illness outcomes 14 effect estimates were reported. Two show inverse associations between measures of social capital and mental illness (cognitive compared with psychosis and combined compared with suicide), two positive associations (structural compared with suicide and combined compared with substance misuse), and 10 no association.

\section{Cognitive}

In contrast with the results from the individual social capital studies, there did not seem to be an inverse association 
Table 2 Summary of data on the association between individual social capital and mental illness

\begin{tabular}{|c|c|c|c|c|}
\hline & \multirow[b]{2}{*}{$\begin{array}{l}\text { Number of effect } \\
\text { estimates }\end{array}$} & \multicolumn{3}{|c|}{ Number of effect estimates (sample size) } \\
\hline & & $\begin{array}{l}\text { Inverse } \\
\text { association }\end{array}$ & No association & $\begin{array}{l}\text { Positive } \\
\text { association }\end{array}$ \\
\hline \multicolumn{5}{|l|}{ Cognitive } \\
\hline Common mental disorder & 11 & $7(18140)$ & $4(2608)$ & 0 \\
\hline Child mental illness & 7 & $2(1008)$ & $5(3429)$ & 0 \\
\hline \multicolumn{5}{|l|}{ Structural } \\
\hline $\begin{array}{l}\text { Common mental disorder } \\
\text { Combined }\end{array}$ & 11 & $3(56971)$ & $7(23832)$ & $1(222)$ \\
\hline $\begin{array}{l}\text { Combined } \\
\text { Common mental disorder }\end{array}$ & 2 & $2(5952)$ & 0 & 0 \\
\hline Total & 31 & & & \\
\hline
\end{tabular}

Table 3 Summary of data on the association between ecological social capital and mental illness

\begin{tabular}{|c|c|c|c|c|}
\hline & \multirow[b]{2}{*}{$\begin{array}{l}\text { Number of effect } \\
\text { estimates }\end{array}$} & \multicolumn{3}{|c|}{ Number of effect estimates (sample size) } \\
\hline & & $\begin{array}{l}\text { Inverse } \\
\text { association }\end{array}$ & No association & $\begin{array}{l}\text { Positive } \\
\text { association }\end{array}$ \\
\hline \multicolumn{5}{|l|}{ Cognitive } \\
\hline Common mental disorder & 4 & 0 & 4 (28369) & 0 \\
\hline Psychotic & 1 & 1 (NS) & 0 & 0 \\
\hline Child mental illness & 2 & 0 & $2(1152)$ & 0 \\
\hline \multicolumn{5}{|l|}{ Structural } \\
\hline Common mental disorder & 1 & 0 & $1(9223)$ & 0 \\
\hline Suicide & 2 & 0 & $1(\mathrm{NS})$ & 1 (NS) \\
\hline \multicolumn{5}{|l|}{ Combined } \\
\hline Psychotic & 1 & 0 & $1(3293)$ & 0 \\
\hline Substance misuse & 2 & 0 & $1(3293)$ & $1(3293)$ \\
\hline Suicide & 1 & $1(121933)$ & 0 & 0 \\
\hline Total & 14 & & & \\
\hline
\end{tabular}

between ecologically measured cognitive social capital and common mental disorders. Similarly, no association was reported by the study investigating cognitive social capital and child mental illness. ${ }^{58}$

An inverse association was reported between the level of cognitive social capital and schizophrenia but this study was a pilot that did not adjust for potential confounders. ${ }^{57}$

\section{Structural}

None of the three effect estimates in this section reported an inverse association between the level of structural social capital and mental illness. One effect estimate reported a higher risk of suicide in areas with higher structural social capital (Harper et al, unpublished data) while the other two showed no association (Stafford ${ }^{56}$ and Harper et al (unpublished data)).

\section{Combined}

Two studies explored the association between combined measures of social capital and mental illness, yielding four effect estimates. One study showed an inverse association with rates of suicide ${ }^{59}$ while the other showed no significant association with psychotic disorders or drug misuse, but showed higher social capital to be associated with increased alcohol problems. ${ }^{60}$

\section{DISCUSSION}

\section{Discussion of results}

The results for individual social capital and ecological social capital measurements are very different. This may underline the assertion that individual and ecological social capital do not measure the same thing, and supports arguments that it may be confusing to consider them as similar. Individual social capital can be considered a measure of the respondent's appraisal of their environment, their social networks, and their level of participation in their community. Depression and anxiety produce characteristic ways of thinking, with more negative appraisal associated with anxiety and depression. In addition, social withdrawal in depression means that sufferers are less likely to play an active part in the community than those who are not. ${ }^{62}$ Hence, it would not be surprising for people with common mental disorders to score low on individual social capital scales.

However, while cognitive social capital (feelings of trust and reciprocity) was shown to be inversely associated with common mental disorders as expected, the varied results of associations between structural social capital (group

\section{What this paper adds}

This paper offers the most comprehensive assessment of the evidence of an association between social capital and mental health to date. It shows that there is strong evidence for an inverse association between individual level cognitive social capital and common mental disorders but few other consistent associations, largely because of the disparate nature of the research conducted to date. 


\section{Policy implications}

Social capital has influenced national mental health strategies around the world and is influencing policy development. This review shows that such policies may be premature as the current evidence is inconclusive.

membership) and common mental disorder may warrant further investigation. For instance, if individual assessments of structural social capital merely reflected the disease process then social withdrawal attributable to anxiety or depression would be expected to be associated with low levels of social capital. This is not what we found. An alternate view, however, could be that in areas of low social cohesion and high employment those with a sick role have more time and opportunities for cross community integration than others. Such forces could work in opposite directions on the association between structural social capital and common mental disorders. There are, of course many more detailed and elaborate possibilities that would need in depth longitudinal studies to unravel.

There was no pattern to the results of studies investigating the association between ecological social capital and mental illness. These studies were small in number and diverse in nature and therefore difficult to compare, however there was no clear evidence for an inverse association between the level of social capital and mental illness.

Of particular interest are the two longitudinal studies that showed contradictory results. One found an inverse association whereby high levels of social capital were associated with a decreased risk of suicide in discharged veterans. ${ }^{59}$ The other showed a positive association whereby homeless mentally ill patients who lived in counties with high social capital had lower rates of recovery from alcohol dependence than patients who lived in counties with low social capital. ${ }^{51}$ Previous research has questioned whether populations with higher social capital would be less tolerant of diversity and more likely to stigmatise the mentally ill. ${ }^{60}$ A unifying hypothesis to explain these results is that in the USA the homeless mentally ill may be stigmatised but veterans considered more deserving of community support.

\section{Methodological limitations of studies}

This review highlights the limitations of research conducted to date and lends credence to the criticism that the current measurement of social capital does not match up to the theory. ${ }^{63}{ }^{64}$ A particularly serious limitation is the predominance of cross sectional studies, which makes the direction of association between social capital and mental illness impossible to determine. It is highly plausible that mental illness could result in low social capital.

While the theory recognises that social capital is a multidimensional concept encompassing both bridging, bonding and linking aspects of social relationships, ${ }^{12}{ }^{64}$ none of the studies included in the review measure any aspect of bridging or linking social capital. Indeed, 15 of the 21 studies measure either only one dimension of social capital (structural or cognitive), or combine these two dimensions into one score.

In addition, three of the seven studies measuring ecological social capital retro-fit concepts of social capital onto existing survey questions rather than developing questions specifically to measure social capital (Harper et al (unpublished data), Desai et $a l^{59}$ and Rosenheck et $\left.a l^{60}\right)$. Although the remainder ask individuals within each community a series of questions that are then aggregated to the community level, no study uses a contextual measure of social capital that does not rely exclusively on individual perceptions of their community. ${ }^{66}$

Although many studies use quantitative techniques such as factor analysis to determine the internal validity of sub-scales of social capital, only one study used a qualitatively piloted and validated measure that explored the external validity of the questions by determining respondents' interpretation of the questions. ${ }^{56}$ The problem of how to validate social capital tools remains one of the important challenges facing this research. ${ }^{1266}$

The criticism that ecological social capital, while attempting to analyse relationships within communities, often uses levels of aggregation that do not reflect respondents' definitions of their community is borne out by this review, as is the paucity of proposed causal mechanisms through which social capital could affect mental illness. In addition, many different levels of aggregation are used, ranging from electoral ward to state level. As in the income inequality literature where different effects have been shown at different levels of aggregation, ${ }^{67}$ the differences between the results of the ecological studies may also be explained by different levels of aggregation measuring different types of social capital.

However, most studies adequately adjusted for confounding by the most important confounder socioeconomic status, and the almost universal use of multilevel modelling among the studies measuring ecological social capital ensured a degree of analytical sophistication.

While urban, and particularly poor urban populations, are well represented by the studies conducted to date, the impact of social capital on rural populations and in the developing world is not well researched.

\section{Limitations of the review}

This review is subject to a number of limitations. Firstly, unpublished studies by research groups who have not already published material about social capital and mental illness may be under-represented.

Secondly, the method of combining results according to a statistical significance level of $5 \%$ is insensitive. Three studies report effect estimates significant at the 10\% level but are classed as non-significant by the analysis. ${ }^{5055}$ Equally, 12 of the effect estimates are significant at the $1 \%$ level or lower, yet this degree of significance could not be shown. Perhaps the greatest problem is the inconsistency of reporting of results by studies. Two do not report coefficients if they are not significant at the $5 \%$ level, and nine studies do not report confidence intervals. This makes it extremely difficult to judge if non-significant results show "no effect" or reflect lack of study power. Lastly, treating each effect estimate as if it is from a different study gives more weight to studies that report more than one exposure or outcome. We did not apply a Bonferroni correction to the significance levels of studies with more than one outcome and thus may have overestimated the significance of coefficients from these studies. This was because while a Bonferroni correction decreases the chance of a type I error this is at the cost of an increased chance of a type II error. In addition, the bias inherent in only selecting one effect estimate from each study was considered greater than that of over-estimating the significance of a few coefficients.

Lastly, it is possible that some studies investigating social support and mental illness measured variables that would satisfy criteria for individual social capital but were not included. Short of repeating existing reviews of the vast social support literature it is difficult to know how this could be avoided. We assessed all papers in which social capital or its synonyms were in a heading, abstract, or keyword. We also searched the reference lists of papers that met the inclusion 
criteria. The identified need of our review was to investigate the evidence for a link between social capital and mental illness that was driving current health strategies and policy initiatives. We believe that our strategy will have captured the available literature.

\section{Conclusion}

This review reflects the current state of the literature on the association between social capital and mental illness. Among the individual social capital studies, strong evidence was found for an inverse association between cognitive social capital and common mental disorders. There was less evidence for an inverse association between cognitive social capital and child mental illness, and combined measures of social capital and common mental disorders. The ecological studies were diverse in methodology, populations investigated, and mental illness outcomes, making them difficult to compare.

The strength of the current evidence, in particular that from studies measuring ecological social capital, is inadequate to inform the need for or development of specific social capital interventions to combat mental illness. A programme of further research is urgently required.

By outlining the state of current research, this review is able to highlight some of the issues that future research should address:

(1) Measure all dimensions of social capital-that is, cognitive, structural, bridging, bonding, and linking

(2) Use validated social capital measures

(3) Be explicit about causal pathways between social capital and mental illness

(4) Examine associations longitudinally

(5) Research in developing world and among rural populations

Specifically for ecological social capital studies:

(1) Use a level of aggregation that reflects respondents' perception of their community if measuring community social capital

(2) Use multilevel modelling when linking ecological social capital to individual outcomes

There is growing interest in the design and implementation of social interventions for the promotion of mental health. Future research on social capital and mental illness should inform the future design of such programmes.

\section{ACKNOWLEDGEMENTS}

We thank Fiona Renton (Cochrane Information Scientist) for providing assistance with the selection of databases and search terms, and Nigel De Silva for comments on earlier drafts. We also thank the many authors who responded with information about unpublished studies or with more information about published studies, and the very helpful comments of reviewers.

\section{Authors' affiliations}

M J De Silva, S R Huttly, Nutrition and Public Health Intervention Research Unit, London School of Hygiene and Tropical Medicine, UK K McKenzie, Department of Psychiatry and Behavioural Sciences, Royal Free and UCL Medical School, London, UK and Barnet Enfield and Haringey Mental Health Trust, UK

T Harpham, Department of Urban Development and Policy, London South Bank University and London School of Hygiene and Tropical Medicine

Funding: Mary De Silva is supported by a studentship from the UK Medical Research Council.

Competing interests: none.

\section{APPENDIX}

\section{LIST OF DATABASES SEARCHED AND SEARCH TERMS USED}

- PubMed

- Embase

- PsychInfo

- IBSS

- Science and Social Science Citation Index

- TRIP Database

- Popline

- $\mathrm{CAB}$ abstracts

- HMIC

- SERFile

- SIGLE4

- Lilacs

- Inter-American initiative on social capital, ethics and development - document library

- World Bank Social Capital document library

- Eldis

- ID21

- National Research register

- Zetoc

- Cochrane Library

- C2-SPECTR

\section{Search terms}

\#mental disorders OR \#psychology OR \#psychiatry OR "mental illness" OR "mental distress" OR "mental health" OR "mental well-being" OR "emotional well-being" OR "psychological well-being"

AND

"social capital" OR "social cohesion" OR "neighbourhood cohesion" OR "neighborhood cohesion" OR "informal social control" OR "collective efficacy" OR "neighbourhood disorder" OR "social disorganization" OR "social disorganisation"

Note $\#=$ exploded meSH term encompassing all mesh sub-headings. Search terms in quotes are searched for as exact text phrases.

\section{REFERENCES}

1 Henderson S, Whiteford $\mathrm{H}$. Social capital and mental health. Lancet 2003;362:505-6.

2 Department of Health. Making it happen a guide to mental health promotion. London: TSO, 2001:46-50.

3 Department of the Deputy Prime Minister. Mental health and social exclusion. London: Social Exclusion Unit, 2004:104-7.

4 Putnam R. Making democracy work: civic traditions in modern Italy. Princeton, NJ: Princeton University Press, 1993.

5 Coleman JS. Social capital in the creation of human capital. American Journal of Sociology 1988;94:95-120.

6 Portes A. Social capital: its origins and applications in modern sociology. Annual Review of Sociology 1998;24:1-24.

7 Bain K, Hicks N. Building social capital and reaching out to excluded groups: the challenge of partnerships. CELAM meeting on the struggle against poverty towards the turn of the millennium, Washington DC, 1998.

8 Szreter S, Woolcock M. Health by association? Social capital, social theory, and the political economy of public health. Int J Epidemiol 2004;33:1-18.

9 McKenzie K, Whitley R, Weich S. Social capital and mental health. Br J Psychiatry 2002;181:280-3.

10 Kawachi I, Berkman LF. Social ties and mental health. J Urban Health 2001;78:458-67.

11 Harris EC, Barraclough B. Suicide as an outcome for mental disorders. Br J Psychiatry 1997; 170:205-28.

12 Harpham T, Grant E, Thomas E. Measuring social capital within health surveys: key issues. Health Policy Plan 2002;17:106-11.

13 Ramirez AJ, Westcombe AM, Burgess CC, et al. Factors predicting delayed presentation of symptomatic breast cancer: a systematic review. Lancet 
14 Albers AB. Poverty, social context and children's mental health across the early life course. Boston: University of Massachusetts, Center for Survey Research, 2001

15 Nation M. Neighborhoods and mental health: a test of the relationship between ecological variables and psychological health. Columbia, SA: University of South Carolina, 2000.

16 Cooper H, Arber S, Fee L, et al. The influence of social support and social capital on health: a review and analysis of British data. London: Health Education Authority, 1999:1-179.

17 Kunovich R, Hodson R. Civil war, social integration and mental health in Croatia. J Health Soc Behav 1999;40:323-43.

18 Rose R. How much does social capital add to individual health? A survey study of Russians. Soc Sci Med 2000;51:1421-35

19 Duncan SC, Duncan TE, Strycker LA. A multilevel analysis of neighborhood context and youth alcohol and drug problems. Prevention Science 2002;3: 125-33

20 Ennett ST, Flewelling RL, Lindrooth RC, et al. School and neighborhood characteristics associated with school rates of alcohol, cigarette, and marijuana use. J Health Soc Behav 1997;38:55-71.

21 Weitzman ER, Kawachi I. Giving means receiving: the protective effect of social capital on binge drinking on college campuses. Am J Public Health 2000;90:1936-9.

22 Parcel TL, Menaghan EG. Family social capital and children's behaviour problems. Soc Psychol Q 1993;56:120-35.

23 Hendryx MS, Ahern MM. Access to mental health services and health sector social capital. Adm Policy Ment Health 2001 ;28:205-18.

24 McKenzie K. Neighbourhood safety and mental health outcomes. http:// wwwworldbankorg/poverty/scapital/sctalk/talk28htm 2000.

25 Hendryx M, Ahern M. Mental health functioning and community problems. Journal of Community Psychology 1997;25:147-57.

26 Kelleher MJ, Daly M. Suicide in Cork and Ireland. Br J Psychiatry 1990; 157:533-8.

27 Souetre $E$, Wehr TA, Douillet $P$, et al. Influence of environmental factors on suicidal behavior. Psychiatry Res 1990;32:253-63.

28 Kalff AC, Kroes $M$, Vles JS, et al. Neighbourhood level and individual level SES effects on child problem behaviour: a multilevel analysis. J Epidemiol Community Health 2001;55:246-50.

29 Driessen G, Gunther N, van Os J. Shared social environment and psychiatric disorder; a multilevel analysis of individual and ecological effects. Soc Psychiatry Psychiatr Epidemiol 1998;33:606-12.

30 Van Os J, Driessen G, Gunther N, et al. Neighbourhood variation in schizophrenia incidence: evidence for person-environment interaction. Br J Psychiatry 2000;176:243-8.

31 Linn JG, Husaini BA, Whitten-Stovall R, et al. Community satisfaction, life stress, social support, and mental health in rural and urban southern black communities. J Community Psychol 1989;17:78-88.

32 Hao L, Johnson RW. Economic, cultural, and social origins of emotional wellbeing: comparisons of immigrants and natives at midlife. Res Aging 2000;22:599-629.

33 LaGory M, Fitzpatrick K. The effects of environmental context on elderly depression. J Aging Health 1992;4:459-79.

34 O'Brien DJ, Hassinger EW, Dershem L. Community attachment and depression among residents in two rural Midwestern communities. Rural Sociology 1994;59:255-65.

35 Pevalin DJ. Intra-household differences in neighbourhood attachment and their associations with health. In: Morgan A, ed. Social capital for health: insights from quantitative research. London: Health Development Agency, 2003.

36 Davidson WB, Cotter PR. The relationship between sense of community and subjective well-being: a first look. J Community Psychol 1991;19:246-53.

37 Schulz A, Williams D, Israel B, et al. Unfair treatment, neighbourhood effects, and mental health in the Detroit Metropolitan area. J Health Soc Behav 2000;41:314-32.

38 Ross CE. Neighbourhood disadvantage and adult depression. J Health Soc Behav 2000;41:177-87.

39 Sooman A, Macintyre S. Health and perceptions of the local environment in socially contrasting neighbourhoods in Glasgow. Health Place 1995; 1:15-26.

40 Macintyre S, Ellaway A. Neighbourhood cohesion and health in socially contrasting neighbourhoods: implications for social exclusion and public health agenda. Health Bull 2000;60:450-6.
41 McCulloch A. Social environments and health: cross-sectional national survey. BMJ 2001;323:208-9.

42 Pevalin D, Rose D. Social capital for health: investigating the links between social capital and health using the British household panel survey. London: Health Development Agency, 2003.

43 Boreham R, Stafford M, Taylor R, eds. Health survey for England 2000: social capital and health. London: The Stationery Office, 2003.

44 Steptoe A, Feldman PJ. Neighborhood problems as sources of chronic stress: development of a measure of neighborhood problems, and associations with socioeconomic status and health. Ann Behav Med $2001 ; 23: 177-85$.

45 Ellaway A, Maclntyre S, Kearns A. Perceptions of place and health in socially contrasting neighbourhoods. Urban Studies 2001;38:2299-316.

46 Runyan DK, Hunter WM, Socolar RRS, et al. Children who prosper in unfavorable environments: the relationship to social capital. Pediatrics 1998;101:12-18.

47 Aneshensel CS, Sucoff CA. The neighborhood context of adolescent mental health. J Health Soc Behav 1996;37:293-310.

48 Ross CE, Reynolds J, Geis K. The contingent meaning of neighbourhood stability for residents psychological well-being. American Sociological Review 2000;65:581-97.

49 Mitchell C, La Gory M. Social capital and mental distress in an impoverished community. City and Community 2002;1:199-222.

50 Brown DR, Gary LE, Greene AD, et al. Patterns of social affiliation as predictors of depressive symptoms among urban blacks. J Health Soc Behav 1992;33:242-53.

51 Dumont KA. Links between three types of neighbourhood conditions and psychological distress among poor, African-American and Latino women in New York City. Newark, NJ: Department of Psychiatry, New Jersey Medical School, 2002

52 Dumont KA. Links between neighbourhood conditions and psychological distress in middle childhood. Newark, NJ: Department of Psychiatry, New Jersey Medical School, 2002

53 O'Brien D, Patsiorkovski V, Dershem L, et al. Household production and symptoms of stress in post-Soviet Russian villages. Rural Sociology 1996:61:674-98

54 Thomas E. Social capital and women's health in sub Saharan Africa. London: South Bank University, University of London, 2003.

55 Harpham T, Grant E, Rodrigues C. Mental health and social capital in Cali, Columbia. Soc Sci Med 2004;58:2267-77.

56 Stafford M, Bartley M, Boreham R, et al. Neighbourhood social cohesion and health: investigating associations and possible mechanisms. In: Morgan A, Swann C, eds. Social capital for health. Issues of definition, measurement and links to health. London: Health Development Agency, 2004.

57 Boydell J, McKenzie K, van Os J. The social causes of schizophrenia: an investigation into the influence of social cohesion and social hostility Schizophr Res 2002;53:264.

58 Drukker M, Kaplan C, Feron F, et al. Children's health-related quality of life, neighbourhood socio-economic deprivation and social capital. A contextual analysis. Soc Sci Med 2003;57:825-41.

59 Desai RA, Dausey DJ, Rosenheck RA. Mental health service delivery and suicide risk: the role of individual and facility factors. Am J Psychiatry 2005:62:31 1-18.

60 Rosenheck R, Morrissey J, Lam J, et al. Service delivery and community: social capital, service systems integration, and outcomes among homeless persons with severe mental illness. Health Serv Res 2001;36:691-710.

61 Cutrona CE, Russell DW, Hessling RM, et al. Direct and moderating effects of community context on the psychological well-being of African American women. J Pers Soc Psychol 2000;79:1088-101

62 Gelder M, Gath D, Mayou R. Oxford textbook of psychiatry. Oxford: Oxford University Press, 1990:217-68

63 Stone W. Measuring social capital. Towards a theoretically informed measurement framework for researching social capital in family and community life. Melbourne: Australian Institute of Family Studies, 2001:1-46.

64 Woolcock M. Social capital and economic development: toward a theoretical synthesis and policy framework. Theory and Society 1998;27:151-208.

65 Lochner K, Kawachi I, Kennedy BP. Social capital: a guide to its measurement. Health and Place 1999:5:259-70.

66 Macinko J, Starfield B. The utility of social capital in research on health determinants. Milbank Q 2001;79:387-427.

67 Wilkinson RG. Comment: income inequality and social cohesion. Am J Public Health 1997;87:1504-6. 